\title{
LIMITING EFFICIENCIES OF NOVEL SOLAR CELL CONCEPTS IN SPACE
}

\author{
A. $\operatorname{Martí}^{(1)}$, A.Datas ${ }^{(1)}$, J.R.González ${ }^{(2)}$, C. Baur ${ }^{(2)}$ \\ (I) Instituto de Energía Solar, Universidad Politécnica de Madrid,Email:amarti@etsit.upm.es \\ (2)European Space Agency Email.Jose.Ramon.Gonzalez@esa.int
}

\begin{abstract}
In this work we study the limiting efficiency in space environmental conditions of three novel solar cell concepts (hot carrier solar cells, multiple exciton generation solar cells and intermediate band solar cells) and how this limiting efficiency is impacted by the temperature and degradation due to radiation. Comparisons are made with state of the art triplejunction solar cells whose performance is taken as reference. In the last section of the work we briefly review the status related to the experimental achievements of these cells to date.
\end{abstract}

\section{NOVEL SOLAR CELL CONCEPTS OVERVIEW}

In order to exceed the limiting efficiency of single gap solar cells, three novel solar cell approaches have been proposed: the hot carrier solar cell (HCSC), the multiple exciton generation solar cell (MEGSC) and the intermediate band solar cell (IBSC).

In the HCSC (Fig. 1a) photons $(h v)$ create electron-holepairs. However, the excited electrons and holes are not allowed to interact with the semiconductor lattice so that they do not cool down and, therefore, they do not lose their energy in thermalization processes with the semiconductor lattice. It is from this property that the HCSC heritages its efficiency advantage over single gap solar cells. The extraction of these hot electrons and holes from the solar cell demands, however, the use of special contacts (named energy selective contacts) in order to make possible that electrons cool down reversibly to the contact temperature at the time they increase their electro-chemical potential. The HCSC was proposed by Ross and Nozik [1] and reviewed by Wuerfel [2,3] who realised of the necessity of the energy selective contacts.

In the multiple exciton generation solar cell (MEGSC, Fig. 1b) high energy photons $(h v)$ create high energy excited electron-hole pairs (or excitons). This pairs do not ideally lose their energy through thermalization processes but, instead, create one or more additional electron-hole pairs $\left(\mathrm{e}_{1}-\mathrm{h}_{1} ; \mathrm{e}_{2}-\mathrm{h}_{2}, \mathrm{e}_{3}-\mathrm{h}_{3}\right)$ depending on how many times their energy exceeds the energy of the gap. The MEGSC, proposed by Nozik [4], is an evolution of the impact ionization solar cell (IISC) proposed by Werner, Brendel and Queisser [5]. The difference relies on the fact that, while the IISC was thought to be implemented in bulk semiconductors, the MEGSC is envisaged to be implemented using quantum dots. By using quantum dots, the probability of one photon creating more than one electron-hole pair is increased because the momentum conservation selection rule is not required.

The intermediate band solar cell (IBSC) is based on the idea of implementing a semiconductor like material that, instead of exhibiting one single gap, it would exhibit two bandgaps (Fig. 1c). The two bandgaps appear when an "intermediate band" (IB) is created inside a conventional high bandgap semiconductor host. This IB allows the absorption of below bandgap energy photons that, in conventional solar cells are wasted. This absorption occurs through the successive promotion of electrons from the valence band (VB) to the IB and from the IB to the conduction band (CB). Thanks to the formation of the additional bandgap, the IBSC is capable of performing, when properly optimized, with an efficiency close to that of a triple junction solar cell [6].

The limiting efficiency of these novel concepts has been calculated in other works for operation on Earth surface and often, for operation for the sun assumed as a black body at 6000 and maximum concentration (46050 suns). However, this limiting efficiency has never been calculated systematically for AM0 [7] to our knowledge. We think this is necessary as a first step to guide the possible application of these novel concepts in space. In addition, when assuming operation on Earth, the solar cell is usually assumed to operate at $300 \mathrm{~K}$. We think that, with operation in space conditions in mind, evaluating the impact of temperature on cell efficiency is also of relevance. Finally, the limiting efficiency of these novel concepts is usually calculated assuming the so-called radiative limit, a concept by which electronhole pairs can only disappear by emitting one photon (radiative recombination). However, in space, solar cell degradation by radiation plays an important role and, in the next sections, we will also explore the impact of this degradation on the performance of these cells. 


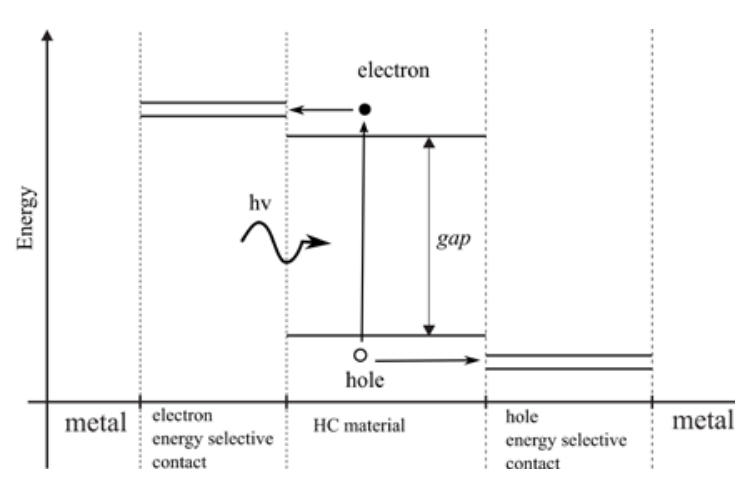

(a)

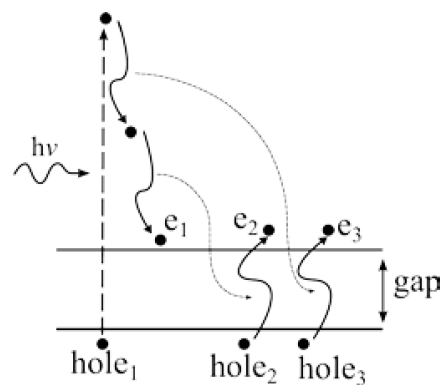

(b)

conduction band

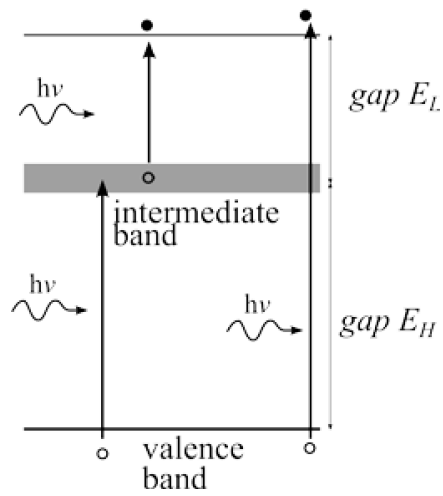

Figure 1. Illustration of the basic structure and operation of (a) a hot carrier solar cell; (b) a multiple exciton generation solar cell and (c) an intermediate band solar cell.

\section{HOT CARRIER SOLAR CELL}

Figure 2 plots the limiting efficiency of the HCSC for AM0 illumination as a function of the semiconductor bandgap. The physical model used to calculate this limiting efficiency is known as "energy conservation model" adapted for AM0 spectral conditions [2]. The impact of the temperature, in the range from $103 \mathrm{~K}$ to $413 \mathrm{~K}$ (minimum temperature has been considered for a standard Geostationary Orbit -GEO- mission; maximum temperature has been considered for a LowEarth Orbit-LEO- mission ) is also illustrated. As it can be seen, the optimum bandgap for operation at $300 \mathrm{~K}$ is located at around $0.9 \mathrm{eV}$ (with a $51.8 \%$ limiting efficiency) and shifts towards higher values (1 eV) when the temperature of operation is increased to $413 \mathrm{~K}$ and towards lower values $(0.6 \mathrm{eV})$ when the temperature is decreased to $103 \mathrm{~K}$. The limiting efficiency decreases from $51.8 \%$ to $41.7 \%$ when the temperature is increased to $413 \mathrm{~K}$ and increases to 76.8 $\%$ when the temperature is decreased from $300 \mathrm{~K}$ to 103 $\mathrm{K}$.

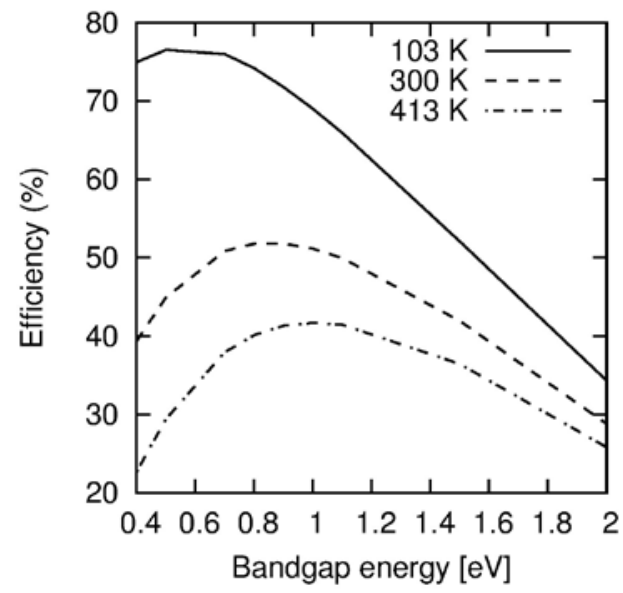

Figure 2. Limiting efficiency of the hot carrier solar cell for AMO illumination conditions as a function of the semiconductor bandgap and the temperature of operation of the cell.

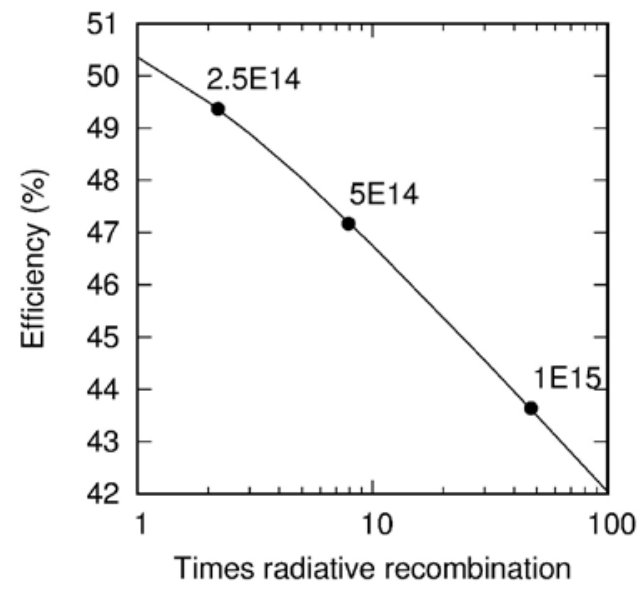

Figure 3. Impact of non-radiative recombination (measured as times the radiative recombination) on the limiting efficiency of the hot carrier solar cell. The temperature for the case illustrated has been assumed $300 \mathrm{~K}$ and the bandgap of the cell $0.9 \mathrm{eV}$ which corresponds to the optimum gap for operation at this temperature.

Figure 3 illustrates the impact of the degradation of the cell due to the appearance of non-radiative recombination measured in times the radiative recombination. In this respect, a factor 5, for example, implies that radiation has introduced non-radiative 
recombination in the cell that is equivalent to 5 times the radiative recombination that is calculated according to detailed balance arguments. As it can be observed, efficiency degrades from $51.8 \%$ to $42.0 \%$ when nonradiative recombination is 100 times the radiative recombination. In order to have a useful meaning in space environment, these factors should be correlated with radiation fluence. However, this is impossible to do, at least while the HCSC remains mostly a theoretical concept since, for example, the materials to implement the concept with have not been clearly identified yet. In this respect, the references to fluence that appear in the Figure refer to fluences that have been estimated to introduce the indicated non-radiative recombination in conventional triple junction solar cells according to the explanations that will be given in Section 5 .

\section{MULTIPLE EXCITON GENERATION SOLAR CELL}

Figure 4 plots the limiting efficiency of the MEGSC for AM0 illumination as a function of the semiconductor bandgap. The physical model used to calculate this limiting efficiency is the one described in [5] adapted for the AM0 spectral conditions. The optimum bandgap for operation at $300 \mathrm{~K}$ is found to be $0.73 \mathrm{eV}$ (with a $43.1 \%$ limiting efficiency), $0.89 \mathrm{eV}$ for $413 \mathrm{~K}$ (with a $33.9 \%$ ) and $0.32 \mathrm{eV}$ for $103 \mathrm{~K}$ (and a limiting efficiency of $68.1 \%$ ).

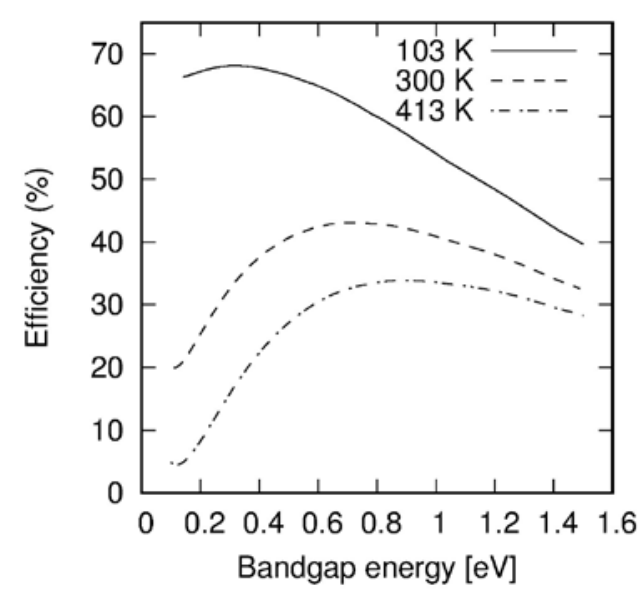

Figure 4. Limiting efficiency of the multiple exciton generation solar cell for AMO illumination conditions as a function of the semiconductor bandgap and the temperature of operation of the cell.

As we did for the HCSC, Figure 5 illustrates now the impact of the appearance of non-radiative recombination in the efficiency of the cell. Fluences producing the same non-radiative recombination in $3 \mathrm{~J}$ MJSC are indicated for reference. As it can be observed, non-radiative recombination seems to have a greater impact on the MEGSC than in the HCSC since, for example, its efficiency degrades below $38 \%$ for a nonradiative recombination 10 times the radiative one while the limiting efficiency of the HCSC remains above 46 $\%$ for the same non-radiative recombination.

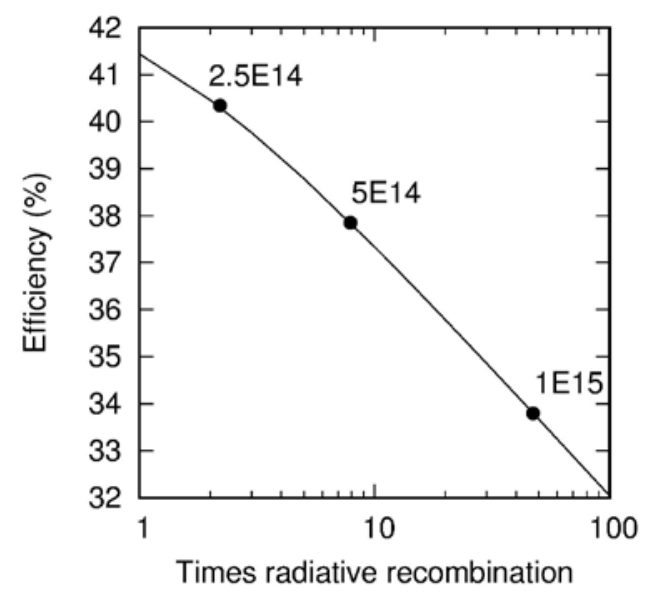

Figure 5. Impact of non-radiative recombination (measured as times the radiative recombination) on the limiting efficiency of the multiple exciton generation cell. The temperature for the case is illustrated has been assumed $300 \mathrm{~K}$ and the bandgap of the cell $0.73 \mathrm{eV}$ which corresponds to the optimum for operation at this temperature.

\section{INTERMEDIATE BAND SOLAR CELL}

As introduced in section 1, the IBSC is characterized by two bandgaps $E_{L}$ and $E_{H}$. Figure 6 plots the limiting efficiency of the IBSC for AM0 illumination as a function of the lowest bandgap $E_{L}$ at the time the value of $E_{H}$ is optimized. The physical model used to calculate this limiting efficiency is the one described in [6] adapted for the AM0 spectral conditions. The optimum bandgap for operation at $300 \mathrm{~K}$ is found to be $E_{L}=0.85$ $\mathrm{eV}$ (with a $45.8 \%$ limiting efficiency and $E_{H}=1.38 \mathrm{eV}$ ). The limiting efficiency degrades to $39 \%$ when the cell operates at $413 \mathrm{~K}$ and increases to $61 \%$ when the temperature decreases down to $103 \mathrm{~K}$. 


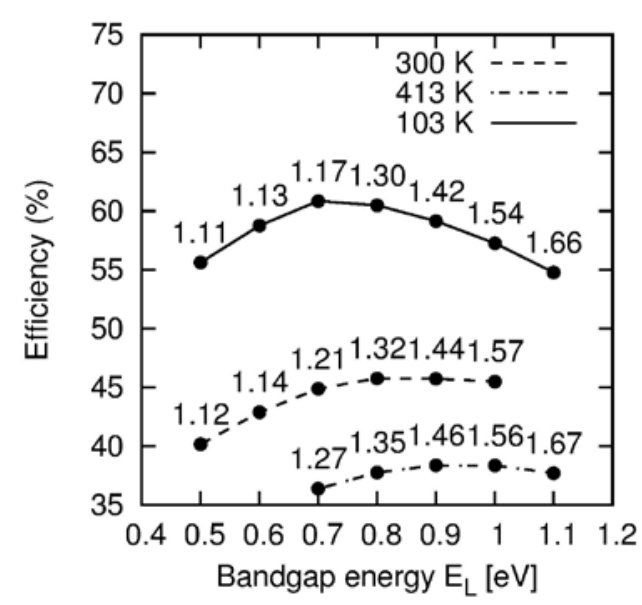

Figure 6. Limiting efficiency of the intermediate band solar for AMO illumination conditions as a function of the semiconductor bandgap $E_{L}$ and the temperature of operation of the cell. Labels indicate the optimum value for $E_{H}$.

Figure 7 shows the impact on the efficiency of the optimum cell $\left(E_{L}=0.85 \mathrm{eV}\right.$ and $\left.E_{H}=1.38 \mathrm{eV}\right)$ for operation at $300 \mathrm{~K}$ of non-radiative recombination. As it can be seen, efficiency would degrade down to $38.5 \%$ when non-radiative recombination becomes one hundred times the radiative one. We indicate in the figure, for reference, the fluences that in the 3J-MJSC are estimated to introduce the same non-radiative recombination

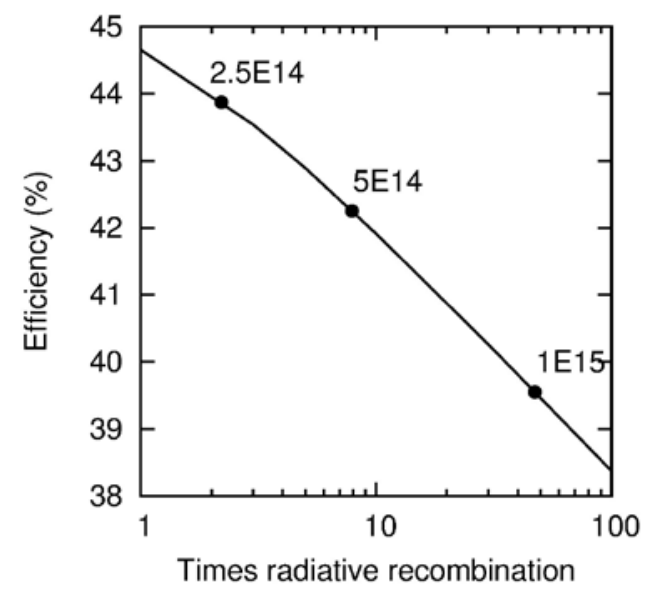

Figure 7. Impact of non-radiative recombination (measured as times the radiative recombination) on the limiting efficiency of the intermediate band solar cell.

The temperature for the case is illustrated has been assumed $300 \mathrm{~K}$ for the bandgaps $E_{L}=0.85 \mathrm{eV}$ and $E_{H}=1.38 \mathrm{eV}$ which corresponds to the optimum gaps for operation at this temperature.

\section{3J-MULTIJUNCTION SOLAR CELL}

It will be illustrative to undertake, for the solar cell commonly used today in space (the GaInP/InGaAs/Ge lattice matched tandem solar cell), the same study we have carried out in the previous sections for the novel solar cell concepts.

In this respect, Figure 8 plots the limiting efficiency of this $3 \mathrm{~J}-\mathrm{MJSC}$ as a function of the temperature. The limiting efficiency has been calculated using detailed balance arguments $[8,9]$ assuming $1.88 \mathrm{eV}, 1,41 \mathrm{eV}$ and $0,66 \mathrm{eV}$ gaps for the GaInP, InGaAs and $\mathrm{Ge}$ respectively. Since due to the lattice matching constrains these gaps are not the ideal ones for a tandem configuration of three solar cells connected in series, a better current matching and efficiency is obtained if the GaInP cell is assumed not to absorb all the sunlight above its bandgap but, instead, $86 \%$ of it while it allows the remaining light to reach the InGaAs middle cell. In contrast, total light absorption has been assumed for the InGaAs and Ge solar cells. No radiative coupling between the cells has been assumed in these calculations.

In the same figure we also plot, for comparison, the dependence of the limiting efficiency with the temperature of the HCSC, MEGSC and IBSC which efficiency was optimum at $300 \mathrm{~K}$. This dependence shows to be linear with the temperature and Table 1 collects this slope for each of the cells. In this respect, the 3J-MJSC shows the lowest dependence $(0.055$ efficiency points of decrease per degree) while the HCSC shows the highest (almost 0.1 points of efficiency per degree).

Table 1 Decrease in efficiency points per $K$ degree for the HCSC, MEGSC and IBSC which operation is optimum at $300 \mathrm{~K}$.

\begin{tabular}{|c|c|c|c|c|}
\hline & 3J-MJSC & HCSC & MEGSC & IBSC \\
\hline $\mathrm{K}^{-1}$ & -0.055 & -0.099 & -0.093 & -0.069 \\
\hline
\end{tabular}

With respect to the degradation induced by nonradiative recombination, Figure 9 plots the limiting efficiency of the 3J-MJSC as a function of the nonradiative recombination. The correlation between "fluence" and the "times radiative recombination" factor advanced in previous sections has been estimated as follows. From public data published by AzurSpace [10] for their commercial triple junction solar cell $3 \mathrm{G} 30 \mathrm{C}-$ Advanced it has been possible to correlate that $1 \mathrm{MeV}$ [e/cm2] fluences of $2.5 \times 10^{14}, 5 \times 10^{14}$ and $10^{15}$ degrade the cell beginning of life (BOL) efficiency by 3.1, 5.8 and $10.2 \%$ respectively. Assuming this degradation percentages in the limiting efficiency of the ideal GaInP/InGaAs/Ge solar cell (Figure 9) leads to non-radiative factors of 2.2, 7.9 and 47.3 respectively. 
These are the factors that we also indicated for reference in the efficiency plots of Figures 3, 5 and 7.

Table 2 summarises the impact on the limiting efficiency of the different novel concepts studied in this work of this levels of non-radiative recombination. As it can be observed, the MEGSC seems to exhibit the highest dependence to non-radiative recombination followed by the HCSC and IBSC.

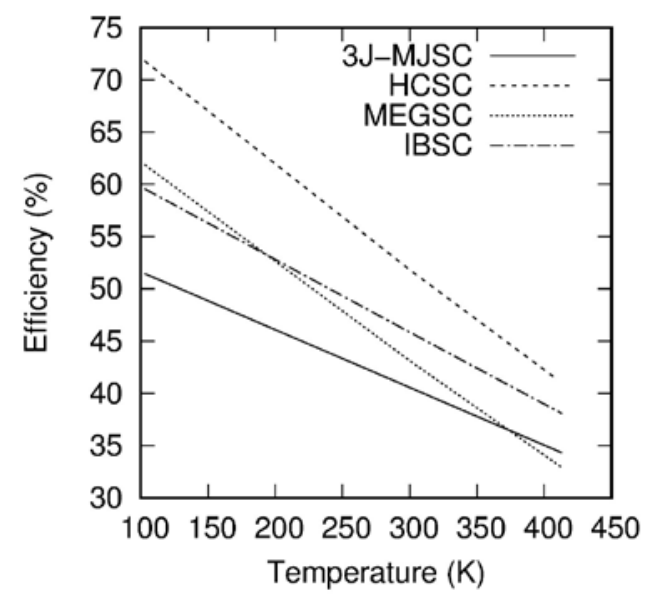

Figure 8. Impact of temperature on the limiting efficiency of the 3J-MJSC (InGaP/InGaAs/Ge lattice matched tandem solar cell), HCSC (0.9 eV), MEGSC $(0,73 \mathrm{eV})$ and IBSC $\left(E_{L}=0.8 \mathrm{eV}\right.$ and $\left.E_{H}=1.32 \mathrm{eV}\right)$. The value of the indicated gaps were found to be optimum for photovoltaic conversion of these cells at $300 \mathrm{~K}$.

Table 2. Limiting efficiency of several novel concepts for several that $1 \mathrm{MeV}[\mathrm{e} / \mathrm{cm} 2]$ fluences. For the calculation we have assumed that the given fluence introduced in the novel concept the same average nonradiative recombination that it would introduce in a $3 \mathrm{~J}$ -

MJSC. For the 3J-MJSC we have assumed a

In GaP/InGaAs/Ge lattice matched solar cell. The

increment $\Delta$ refer to the variation of the efficiency

between the $1 \times 10^{15}$ fluence and the BOL efficiency,

\begin{tabular}{|l|c|c|c|c|c|}
\hline & BOL & $2.5 \times 10^{14}$ & $5 \times 10^{14}$ & $1 \times 10^{15}$ & $\Delta$ \\
\hline HCSC & 51.8 & 49.4 & 71.2 & 43.6 & $\begin{array}{c}-15.8 \\
\%\end{array}$ \\
\hline $\begin{array}{l}\text { MEGS } \\
\text { C }\end{array}$ & 43.1 & 40.3 & 37.9 & 33.8 & $\begin{array}{c}-21.6 \\
\%\end{array}$ \\
\hline IBSC & 45.8 & 43.9 & 42.3 & 39.6 & $\begin{array}{c}-13.5 \\
\%\end{array}$ \\
\hline $\begin{array}{l}\text { 3J- } \\
\text { MJSC }\end{array}$ & 41.8 & 40.5 & 39.4 & 37.5 & $\begin{array}{c}-10.3 \\
\%\end{array}$ \\
\hline
\end{tabular}

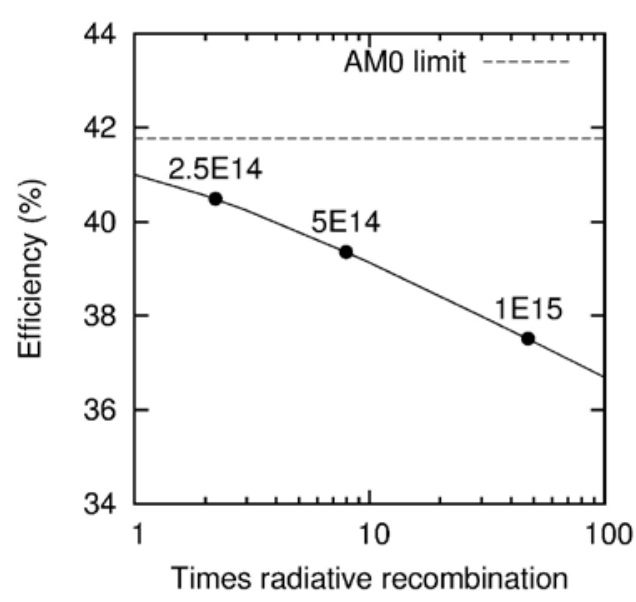

Figure 9. Impact of non-radiative recombination in the limiting efficiency of a 3J-MJSC InGaP/InGaAs/Ge lattice matched tandem solar cell. Dots indicate the 1 $\mathrm{MeV}[\mathrm{e} / \mathrm{cm} 2]$ fluence that is estimated to induce the corresponding non-radiative recombination. (3 gaps in series: $0.7 \mathrm{eV}, 1.1 \mathrm{eV}$ and $1.7 \mathrm{eV}$ ).

\section{BRIEF REVIEW OF THE STATUS OF EMPIRICAL RESEARCH ON NOVEL CONCPETS}

The results above show the limiting efficiency of the HCSC, MEGSC and IBSC for space operation conditions as well as the impact of the temperature and degradation by non-radiative recombination. However, looking forward the real use of these cells, it must be taken into account that, so far, none of these cells have demonstrated the promised efficiencies although, to more or less extent the physical principles involved in their operation have been demonstrated.

Hence, for the case of the IBSC, the absorption of two below bandgap energy photons to create one electronhole pair has been demonstrated, for example, in several implementations that use III-V semiconductor quantum dots [11-15]. In addition, it has also been proved that the presence of the intermediate band does not limit the output voltage of the cell $[16,17]$.

In the MEGSC context, Semonin et al. [18] have demonstrated a solar cell based on colloidal quantum dots that exhibited an external quantum efficiency higher than one.

Research on HCSC focuses, on one side, on finding a suitable hot carrier material and, on the other side, on finding suitable energy selective contacts. As for the hot carrier material concerns, Conibeer et al. have proposed 
[19] materials such as InN, HfN and SnSi. Yao and Köning have proposed BSb [20]. As for the energy selective contacts, the most studied option is based on the exploitation of different variations of the resonant tunnelling using nanostructures [21]. Dimmock et al. [22] have proposed and investigated a HCSC implemented on a metal-semiconductor junction.

\section{CONCLUSIONS}

HCSC, MEGSC and IBSC promise efficiencies of 51.8 $\%, 43.1 \%$ and $45.8 \%$ in ideal conditions for AM0 illumination and $300 \mathrm{~K}$. Regarding the dependence of these limiting efficiencies with temperature, the HCSC has revealed the highest dependence and the IBSC the lowest (see Table I).

As for the impact of non-radiative recombination in this efficiency, assuming this would introduce the same nonradiative recombination factor than in a $3 \mathrm{~J}-\mathrm{MJSC}$, the MEGSC has exhibited the highest sensibility to nonradiative recombination while the IBSC has exhibited the lowest (see Table II).

In the laboratory, however, no cell has demonstrated efficiencies exceeding the efficiency of single gap solar cells. Therefore, their practical application in space will still need to wait a few years more. In spite of this, all the concepts have provided strong experimental support demonstrating that the physical principles they are based on are correct. It is also noticeable that the three concepts demand the use of nanostructures, in a way or another, for their practical implementation.

\section{ACKNOWLEDGMENTS}

This work has been funded by the European Space Agency through the Contract No. 4000115109/15/NL/FE

\section{REFERENCES}

1. Ross, R.T., and Nozik, A.J. (1982). Efficiency of hot-carrier solar energy converters. Journal of Applied Physics 53, 3813-3818.

2. Würfel, P., Brown, A.S., Humphrey, T.E., and Green, M.A. (2005). Particle conservation in the hot-carrier solar cell. Progress in Photovoltaics: Research and Applications 13, 277-285.

3. Wurfel, P. (1997). Solar energy conversion with hot electrons from impact ionisation. Solar Energy Materials and Solar Cells 46, 43-52.

4. Nozik, A.J. (2002). Quantum dot solar cells Physica E 14, 115-120.
5. Jiirgen H. Werner, Rolf Brendel, and Queisser, H.J. (1994). New Upper Efficiency Limits for semiconductor solar cells. Proceedings of the First World Conference on Photovoltaic Energy Conversion.

6. Luque, A., and Martí, A. (1997). Increasing the efficiency of ideal solar cells by photon induced transitions at intermediate levels. Physical Review Letters 78, 5014-5017.

7. 2000 ASTM Standard Extraterrestrial Spectrum Reference E-490-00.

8. Shockley, W., and Queisser, H.J. (1961). Detailed Balance Limit of Efficiency of p-n Junction Solar Cells. Journal of Applied Physics 32, 510-519.

9. Martí, A., and Araújo, G.L. (1996). Limiting efficiencies for photovoltaic energy conversion in multigap systems Solar Energy Materials and Solar cells 43, 203-222.

10. AzurSpace

URL:http://www.azurspace.com/index.php/en/pro ducts/products-space/space-solar-cells. Accessed: 2016-06-02. (Archived by WebCite ${ }^{\circledR}$ at http://www.webcitation.org/6hxzSFIDP).

11. Martí, A., Antolin, E., Stanley, C.R., Farmer, C.D., Lopez, N., Diaz, P., Canovas, E., Linares, P.G., and Luque, A. (2006). Production of Photocurrent due to Intermediate-to-ConductionBand Transitions: A Demonstration of a Key Operating Principle of the Intermediate-Band Solar Cell. Physical Review Letters 97, 247701247704.

12. López, E., Datas, A., Ramiro, I., Linares, P.G., Antolín, E., Artacho, I., Martí, A., Luque, A., Shoji, Y., Sogabe, T., et al. (2016). Demonstration of the operation principles of intermediate band solar cells at room temperature. Solar Energy Materials and Solar Cells 149, 15-18.

13. Datas, A., López, E., Ramiro, I., Antolín, E., Martí, A., Luque, A., Tamaki, R., Shoji, Y., Sogabe, T., and Okada, Y. (2015). Intermediate Band Solar Cell with Extreme Broadband Spectrum Quantum Efficiency. Physical Review Letters $114,157701$.

14. Tamaki, R., Shoji, Y., Okada, Y., and Miyano, K. (2014). Spectrally resolved intraband transitions on two-step photon absorption in InGaAs/GaAs quantum dot solar cell. Applied Physics Letters 105, -. 
15. Iñigo Ramiro , Elisa Antolín, Jinyoung Hwang, Alan Teran, Andy Martin, Joanna Millunchick, Jamie Phillips, Martí, A., and Luque, A. (2016). Three-Bandgap Absolute Quantum Efficiency in Intermediate Band Solar Cells. To appear published at the 43 IEEE PVSC.

16. Linares, P.G., Martí, A., Antolín, E., Farmer, C.D., Ramiro, I., Stanley, C.R., and Luque, A. (2012). Voltage recovery in intermediate band solar cells. Solar Energy Materials and Solar cells 98, 240244.

17. Ramiro, I., Antolin, E., Linares, P.G., Lopez, E., Artacho, I., Datas, A., Marti, A., Luque, A., Steer, M.J., and Stanley, C.R. (2014). Two-photon photocurrent and voltage up-conversion in a quantum dot intermediate band solar cell. In Photovoltaic Specialist Conference (PVSC), 2014 IEEE 40th. pp. 3251-3253.

18. Semonin, O.E., Luther, J.M., Choi, S., Chen, H.Y., Gao, J., Nozik, A.J., and Beard, M.C. (2011). Peak External Photocurrent Quantum Efficiency Exceeding $100 \%$ via MEG in a Quantum Dot Solar Cell. Science 334, 1530-1533.

19. Conibeer, G., Shrestha, S., Huang, S.J., Patterson, R., Xia, H.Z., Feng, Y., Zhang, P.F., Gupta, N., Tayebjee, M., Smyth, S., et al. (2015). Hot carrier solar cell absorber prerequisites and candidate material systems. Solar Energy Materials and Solar Cells 135, 124-129.

20. Yao, Y., and König, D. (2015). Comparison of bulk material candidates for hot carrier absorber. Solar Energy Materials and Solar Cells 140, 422427.

21. Dimmock, J.A.R., Day, S., Kauer, M., Smith, K., and Heffernan, J. (2014). Demonstration of a hotcarrier photovoltaic cell. Prog. Photovoltaics 22, 151-160.

22. Dimmock, J.A.R., Kauer, M., Stavrinou, P.N., and Ekins-Daukes, N.J. (2015). A metallic hot carrier photovoltaic cell. In Physics Simulation and Photonic Engineering of Photovoltaic Devices Iv, Volume 9358, A. Freundlich, J.F. Guillemoles and M. Sugiyama, eds. 